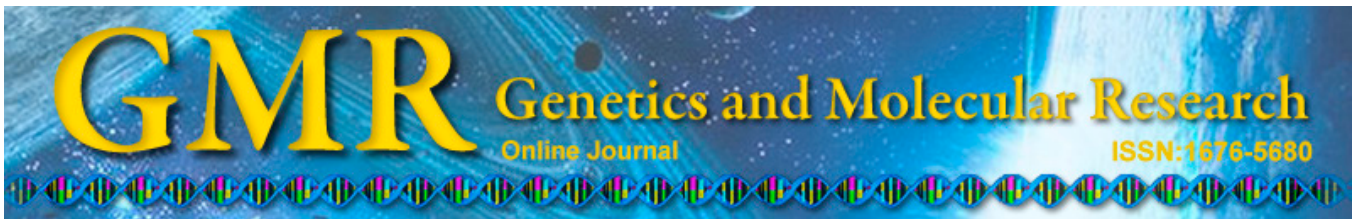

\title{
Transferability of microsatellite markers of Capsicum annuum L. to C. frutescens L. and C. chinense Jacq.
}

S.I.C. Carvalho ${ }^{1,2}$, C.F. Ragassi ${ }^{2}$, I.B. Oliveira ${ }^{3}$, Z.P.S. Amaral ${ }^{4}$, F.J.B. Reifschneider ${ }^{5}$, F.G. Faleiro ${ }^{6}$ and G.S.C. Buso ${ }^{4}$

${ }^{1}$ Faculdade de Agronomia e Medicina Veterinária, Universidade de Brasília, Brasília, DF, Brasil

${ }^{2}$ Embrapa Hortaliças, Brasília, DF, Brasil

${ }^{3}$ Universidade Federal de Goiás, Goiânia, GO, Brasil

${ }^{4}$ Embrapa Recursos Genéticos e Biotecnologia, Brasília, DF, Brasil

${ }^{5}$ Empresa Brasileira de Pesquisa Agropecuária, Brasília, DF, Brasil

${ }^{6}$ Embrapa Cerrados, Brasília, DF, Brasil

Corresponding author: C.F. Ragassi

E-mail: carlos.ragassi@embrapa.br / cragassi@gmail.com

Genet. Mol. Res. 14 (3): 7937-7946 (2015)

Received August 13, 2014

Accepted February 5, 2015

Published July 17, 2015

DOI http://dx.doi.org/10.4238/2015.July.17.1

ABSTRACT. In order to support further genetic, diversity, and phy-
logeny studies of Capsicum species, the transferability of a Capsicum
annuum L. simple sequence repeat (SSR) microsatellite set was ana-
lyzed for $C$. frutescens L. ("malagueta" and "tabasco" peppers) and C.
chinense Jacq. (smell peppers, among other types). A total of $185 \mathrm{SSR}$
primers were evaluated in 12 accessions from 115 C. frutescens $\mathrm{L}$. and
480 C. chinense Jacq, representing different types within each species.
Transferability to C. frutescens $\mathrm{L}$. and C. chinense Jacq. occurred for
116 primers $(62.7 \%)$. Nineteen $(16.37 \%)$ were polymorphic in $C$. frute-
scens $\mathrm{L}$. and $36(31.03 \%$ ) in $C$. chinense Jacq., 17 of which were coin-
cident and could be used to analyze samples obtained for the 2 species.
Among these primers, CA49 showed a different amplitude range of al-
leles between the 2 species ( $130-132$ base pairs for $C$. frutescens $\mathrm{L}$. and 
120-128 base pairs for $C$. chinense Jacq.), and could differentiate the species. A total of 55 alleles were identified among the 19 polymorphic SSR loci among accessions of $C$. frutescens L., with the number of alleles per locus ranging from 2 to 5 , a mean of 2.89 , and the polymorphic information content ranging from 0.30 to 0.65 . The number of alleles identified in $C$. chinense Jacq. was 119 , ranging from 2 to 5 alleles per locus, an average of 3.30, and polymorphic information content from 0.19 to 0.68 . The $C$. annuum L. SSR primers were most often transferable and polymorphic for $C$. frutescens $\mathrm{L}$. and $C$. chinense Jacq., and we present a set of SSR for each species.

Key words: Breeding; Germplasm bank; Pepper; Polymorphism; Simple sequence repeat

\section{INTRODUCTION}

Peppers (Capsicum) are part of the cultural wealth and valuable genetic heritage of the Brazilian biodiversity (Reifschneider et al., 2000). They are grown throughout the country and they include a variety of types, names, sizes, colors, flavors, and pungency. Peppers are cultivated by small, medium, and large producers by family farmers and smallholder agribusiness as an alternative income source. The annual cultivated area for peppers in Brazil is approximately 5000 hectares, yielding approximately $75,000 \mathrm{Mg}$. The average yield depends on the type of pepper, ranging from $10-30 \mathrm{Mg} / \mathrm{ha}$ (Ribeiro et al., 2008).

The genus Capsicum includes approximately 35 taxa (species and varieties) that are grouped into 3 categories according to the level of domestication, corresponding to 5 domesticated and 30 wild taxa (Bianchetti and Carvalho, 2005). Domesticated species include Capsicum chinense Jacq., C. frutescens L., C. annuum L., C. pubescens Ruiz et Pav, and $C$. baccatum L. (Moses et al., 2014). Among these, C. annuum L., C. chinense Jacq. and C. frutescens L. have traditionally been treated as a species complex because of the high genetic and morphological closeness and they are often treated as the same species based on immediate ancestor identification (Pickersgill, 1988). Thus, $C$. chinense Jacq. is easily confused with $C$. frutescens $\mathrm{L}$. accessions; the main morphological distinction between them is the presence of calyx annular constriction, found only in the fruits of $C$. chinense Jacq. (Carvalho et al., 2006).

The fruits of $C$. frutescens $\mathrm{L}$. are generally small, upright, spindle-shaped, red-colored at the mature stage, have very thin walls, are spicy, and the calyx does not have annular constriction. C. frutescens $\mathrm{L}$. also presents much lower morphological variability than other domesticated species (DeWitt and Bosland, 2009). The most common types of this species include the "malaguetas" in Brazil and tabascos in the United States.

C. chinense Jacq. presents great variability expressed in the diversity of shapes and colors of fruits, which ranges from light yellow, strong yellow, orange, salmon, to red and are typically very spicy and aromatic. There are many types in this species, with the best known including the smell peppers, Murupi, goat, cumari of Pará, small beak, and Habanero (Ribeiro et al., 2008).

In the characterization of the Capsicum Germplasm Bank (BG Capsicum) of Embrapa, some C. chinense Jacq. taxonomic classifications were unresolved, as they presented fruit traits typical of $C$. frutescens $\mathrm{L}$., despite presenting the calyx annular constriction, a major distinctive trait of $C$. chinense Jacq. Thus, molecular tools should be used to achieve more accurate classification.

Among the different molecular markers, simple sequence repeat (SSR) microsatellites 
are suitable for detailed genomic analysis. However, for the amplification of SSR loci, knowledge of its DNA sequence is required, implying that the development of specific primers for this type of analysis is a costly and time-consuming process (Zucchi et al., 2003). However, several studies indicate similarity in DNA sequences located in regions at the edges of repetitive sequences, constituents of SSRs, among different species (Ciampi et al., 2008), indicating that these SSR primers can be transferred between related species. This greatly reduces the cost and time required for research (Lorieux et al., 2000).

Several studies have shown high transferability of SSR markers for species within the same genus, including Cucumis (Ritschel et al., 2004), Arachis (Bravo et al., 2006), and even for Capsicum (Yi et al., 2006; Nagy et al., 2007; Ince et al., 2010a). However, the success of this approach depends on the species considered, as the probability of obtaining transferability is inversely proportional to the evolutionary distance among them (Cota et al., 2012).

In this study, we examined the transferability of a C. annuum L. set of primers (Buso et al., 2000) for $C$. frutescens $\mathrm{L}$. and $C$. chinense Jacq. to correctly identify accessions from the 2 species using materials stored in the BG Capsicum of Embrapa to contribute to the conservation and use of this germplasm.

\section{MATERIAL AND METHODS}

\section{Plant material}

Twelve accessions representing different types within each species (Table 1) were selected from a collection of 115 accessions of $C$. frutescens L. and 480 C. chinense Jacq. of the BG Capsicum of Embrapa, based on morphological characterization made by applying 5 internationally recommended descriptors for Capsicum fruits (IPGRI, 1995): color at mature stage, shape, position, calyx annular constriction, and wall thickness. The genotypes studied included 4 accessions of $C$. frutescens $\mathrm{L}$. and 8 accessions of $C$. chinense Jacq.

Table 1. Fruit traits of accessions representing different types within the species Capsicum frutescens $\mathrm{L}$. and C. chinense Jacq. from the germplasm bank of Embrapa. Brasília, DF, 2014.

\begin{tabular}{|c|c|c|c|c|c|c|c|}
\hline \multirow[t]{2}{*}{ Accession $\mathrm{CNPH} n^{\circ}$} & \multirow[t]{2}{*}{ Species } & \multirow[t]{2}{*}{ Type } & \multicolumn{5}{|c|}{ Fruit } \\
\hline & & & $\mathrm{CM}^{\mathrm{a}}$ & Position & Format & $\mathrm{EP}^{\mathrm{b}}$ & $\mathrm{PCA}^{\mathrm{c}}$ \\
\hline 3891 & C. frutescens & malagueta & orange & standing & oblong & 0.4 & no \\
\hline 4266 & C. frutescens & tabasco & red & standing & oblong & 0.3 & no \\
\hline 4353 & C. frutescens & malaguetinha & red & standing & oblong & 0.5 & no \\
\hline 4364 & C. frutescens & malagueta & red & standing & oblong & 0.6 & no \\
\hline 4361 & C. chinense & malagueta & orange & standing & oblong & 0.9 & yes \\
\hline $4325 \mathrm{~A}$ & C. chinense & similar to malagueta & red & standing & oblong & 0.8 & yes \\
\hline 4315 & C. chinense & olho de peixe & red & standing & campanulate & 0.5 & yes \\
\hline 4316 & C. chinense & similar to malagueta & orange & pendulous & campanulate & 1.8 & yes \\
\hline 4327 & C. chinense & similar to habanero & orange & pendulous & triangular & 2.9 & yes \\
\hline $4328 \mathrm{~A}$ & C. chinense & similar to bode & red & pendulous & rectangular & 3 & yes \\
\hline 4332 & C. chinense & similar to cayenne & red & pendulous & oblong & 2.6 & yes \\
\hline 4360 & C. chinense & murupi & yellow & pendulous & oblong & 1.2 & yes \\
\hline
\end{tabular}

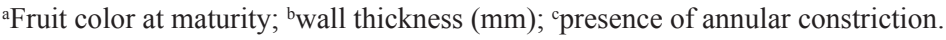

Leaves of each accession were collected and genomic DNA was extracted using the cetyltrimethylammonium bromide method, with some modifications (Ferreira and Grattapaglia, 1998). DNA concentration was estimated by electrophoresis on a $1.0 \%(\mathrm{w} / \mathrm{v})$ agarose gel stained with ethidium bromide; fluorescence intensities between each DNA were compared 
to different patterns of the lambda DNA sample. Each sample was then diluted to $3.0 \mathrm{ng} / \mu \mathrm{L}$.

\section{Isolation of genomic SSRs from $C$. annuum $\mathrm{L}$.}

Approximately $50 \mu \mathrm{g}$ genomic DNA from an individual $C$. annuum L. plant was digested with Tsp509 I, and 280-800-bp fragments were recovered. Approximately $30 \mu \mathrm{g}$ DNA fragments were ligated to adapters containing the Tsp509 I restriction site. The fragments containing SSR sequences were selected by hybridization with biotinylated oligonucleotides complementary to the repetitive sequence $\mathrm{AG} / \mathrm{CT}$, and they were recovered using magnetic beads linked to streptavidin. Recombinant clones in plasmid vectors pGEM-T (Stratagene, La Jolla, CA, USA) were transformed into Escherichia coli XL1-Blue cells, and colonies containing SSRs were identified by polyAG/TC probe hybridization and anchor polymerase chain reaction (PCR). Positive clones were picked and sequenced using an ABI 377 DNA sequencer (Applied Biosystems, Foster City, CA, USA) using dye terminator fluorescent chemistry. Primers annealing at the SSR flanking regions were designed using the software primer (Lincoln et al., 1991).

\section{Primer screening and PCR}

Microsatellite amplification was carried out in a $13-\mu \mathrm{L}$ reaction mix containing 0.9 $\mu \mathrm{M}$ of each primer, $1 \mathrm{U}$ Taq DNA polymerase, $200 \mu \mathrm{M}$ of each dNTP, $1 \%$ (v/v) reaction buffer (10 mM Tris-HCl, $\left.\mathrm{pH} 8.3,50 \mathrm{mM} \mathrm{KCl}, 1.5 \mathrm{mM} \mathrm{MgCl}{ }_{2}\right) 1.3 \mu \mathrm{L} 2.5 \mathrm{mg} / \mathrm{mL}$ bovine serum albumin, and $10.0 \mathrm{ng}$ template DNA. Amplification was performed using a PT-100 thermal controller (MJ Research, Waltham, MA, USA) under the following conditions: 2 min $96^{\circ} \mathrm{C}(1$ cycle), $1 \mathrm{~min} 94^{\circ} \mathrm{C}, 1 \mathrm{~min} 56^{\circ} \mathrm{C}, 1 \mathrm{~min} 72^{\circ} \mathrm{C}(30$ cycles $)$; and $7 \min 72^{\circ} \mathrm{C}(1$ cycle $)$. Each locus was checked for clear and robust DNA band amplification as well as for product polymorphism. Analysis of amplified fragments was carried out on $1.5 \%(\mathrm{w} / \mathrm{v})$ agarose gels stained with ethidium bromide and sized by comparison to a 1-kb DNA ladder standard. Next, PCR with primers that amplified visible products were evaluated on a $5 \%(\mathrm{w} / \mathrm{v})$ polyacrylamide gel stained with silver nitrate following the protocol of Creste et al. (2001).

\section{Data analysis}

A total of 185 microsatellite primers of $C$. annuиm L. were evaluated. Band-size (alleles) evaluation was carried out with reference to the standard size 10-bp DNA ladder. Bands and their respective sizes were recorded in a spreadsheet and analyzed using the Summary Function of Power Marker statistics software (Liu and Muse, 2005), which accounted for sample size, number of observations (equivalent to the number of genotypes evaluated, subtracted from the number of missing genotypes), number of available genotypes (relative to samples not considered), allele number, frequency of the most abundant allele, gene diversity (probability that 2 randomly chosen alleles were different, also referred to as the index of expected heterozygosity), heterozygosity (observed heterozygosity; proportion of heterozygous individuals in a population), and polymorphic information content (PIC, a measure related to gene diversity) for each locus.

\section{RESULTS}

Transferability occurred to $C$. frutescens L. and C. chinense Jacq. for $116(62.7 \%)$ of the 185 SSR primers of $C$. annuиm L. (CA) analyzed. Microsatellite primers designed for $C$. 
annuиm L. (Buso et al., 2000) were, therefore, most often transferable to the 2 species. Among the transferable primers, $19(16.3 \%)$ were polymorphic in C. frutescens L. (Table 2) and 36 (31.0\%) showed polymorphisms for C. chinense Jacq. (Table 3).

Table 2. Primers transferred from Capsicum annuum L. to C. frutescens L. with their motif, sequences of forward and reverse primers, allele number, size range of amplified fragment in base pairs (bp), gene diversity or expected heterozygosity $\left(H_{\mathrm{E}}\right)$, observed heterozygosity $\left(H_{\mathrm{O}}\right)$, and polymorphism information content (PIC). Brasília, DF, 2014.

\begin{tabular}{|c|c|c|c|c|c|c|c|}
\hline Primers (CA) & Motif & $\begin{array}{l}\text { Forward primer/ } \\
\text { reverse primer }\left(5^{\prime}-3^{\prime}\right)\end{array}$ & $\begin{array}{l}\text { Allele } \\
\text { No. }\end{array}$ & $\begin{array}{l}\text { Size range } \\
\text { (bp) }\end{array}$ & $\begin{array}{c}\text { Gene } \\
\text { diversity }\left(H_{\mathrm{E}}\right)\end{array}$ & $\begin{array}{l}\text { Heterozygosity } \\
\left(H_{\mathrm{O}}\right)\end{array}$ & PIC \\
\hline 19 & $(\mathrm{TC})_{12}$ & $\begin{array}{l}\text { CCGCAATGGCAGTATGATCT/ } \\
\text { CGGCTCTATCTACAACGGTG }\end{array}$ & 4 & 98-104 & 0.69 & 1.00 & 0.63 \\
\hline 20 & $(\mathrm{AG})_{27}$ & $\begin{array}{l}\text { CCGTAAAGAAATCAAACCAC/ } \\
\text { GCATGCACACATAAACACTC }\end{array}$ & 3 & $68-88$ & 0.63 & 0.00 & 0.55 \\
\hline 26 & $(\mathrm{AG})_{23}$ & $\begin{array}{l}\text { CGCATATAGGCAGATCAAAT/ } \\
\text { TGACTCAAATGCTCTCTGAA }\end{array}$ & 5 & $100-124$ & 0.69 & 0.75 & 0.65 \\
\hline 27 & $(\mathrm{CA})_{12}(\mathrm{CT})_{17} \mathrm{ATCG}(\mathrm{CT})_{9}$ & $\begin{array}{l}\text { GCAGAGGACCAGTTAGCATA/ } \\
\text { TGTTCTGAGTCCACGATGCT }\end{array}$ & 3 & $128-132$ & 0.63 & 0.50 & 0.55 \\
\hline 29 & $(\mathrm{AG})_{21}$ & $\begin{array}{l}\text { TGGGTAAAGGTACTTAGTAG/ } \\
\text { GTTGTATTGCTTTAGCTCAG }\end{array}$ & 2 & $114-126$ & 0.50 & 0.00 & 0.38 \\
\hline 39 & $(\mathrm{CT})_{13}$ & $\begin{array}{l}\text { CATCCATATATCGATCGGCT/ } \\
\text { TTTCGACCATGTTCAGATCC }\end{array}$ & 3 & 88-98 & 0.63 & 0.00 & 0.55 \\
\hline 41 & $(\mathrm{AG})_{27}$ & $\begin{array}{l}\text { GACATCAGTGTTCTGCAAA/ } \\
\text { ACACTGGGTATGTTGTTGTA }\end{array}$ & 3 & $152-172$ & 0.63 & 0.00 & 0.55 \\
\hline 49 & $(\mathrm{AG})_{21}$ & $\begin{array}{l}\text { CTATCTTCGCATATAGGCAG/ } \\
\text { AATCTCTGTGGCTGACTCAA }\end{array}$ & 2 & $130-132$ & 0.38 & 0.00 & 0.30 \\
\hline 52 & $(\mathrm{GT})_{14}(\mathrm{AG})_{14} \mathrm{TAGC}(\mathrm{GA})_{10}$ & $\begin{array}{l}\text { TAGCAGAGGACCAGTTAGCA/ } \\
\text { ATGTTCTGAGTCCACGATGC }\end{array}$ & 3 & $114-130$ & 0.59 & 1.00 & 0.51 \\
\hline 56 & $(\mathrm{AG})_{23}$ & $\begin{array}{l}\text { CTTCGCATATAGGCAGATCA/ } \\
\text { TCTCTGTGGCTGACTCAAAT }\end{array}$ & 3 & $110-130$ & 0.41 & 0.50 & 0.37 \\
\hline 62 & $(\mathrm{AG})_{22}$ & $\begin{array}{l}\text { CGCATATAGGCAGATCAAAT/ } \\
\text { GGTCAGACTACGCTCTCTCA }\end{array}$ & 4 & $78-84$ & 0.66 & 0.50 & 0.60 \\
\hline 79 & $(\mathrm{TC})_{26}$ & $\begin{array}{l}\text { CACTGGGTATGTTGTTGTAA/ } \\
\text { CCGTAAAGAAATCATACCAC }\end{array}$ & 3 & $90-110$ & 0.63 & 0.00 & 0.55 \\
\hline 88 & $(\mathrm{AG})_{22}$ & $\begin{array}{l}\text { AATGGATGTTCCCTTGCTTT/ } \\
\text { CAACTGATCAACCATTCCGT }\end{array}$ & 2 & $160-162$ & 0.38 & 0.00 & 0.30 \\
\hline 96 & $(\mathrm{AG})_{23}$ & $\begin{array}{l}\text { CGCATATAGGCAGATCAAAT/ } \\
\text { AATCTCTGTGGCTGACTCAAA }\end{array}$ & 3 & $108-116$ & 0.59 & 1.00 & 0.51 \\
\hline 159 & $(\mathrm{AG})_{19}$ & $\begin{array}{l}\text { GCAGAAAATGGGTAAAGGTA/ } \\
\text { ATTGCTTTAGCTCAGAATGG }\end{array}$ & 3 & $110-120$ & 0.63 & 0.50 & 0.55 \\
\hline 167 & $(\mathrm{TC})_{24}$ & $\begin{array}{l}\text { CATTCTCTTCTTCTCAAACG/ } \\
\text { CACGTCAATCTGTTGTGAAA }\end{array}$ & 2 & $80-84$ & 0.38 & 0.00 & 0.30 \\
\hline 172 & $(\mathrm{TC})_{9} \mathrm{GCTA}(\mathrm{TC})_{14}(\mathrm{CA})_{13}$ & $\begin{array}{l}\text { ATGTTCTGAGTCCACGATGC/ } \\
\text { CTTAGCAGAGGGCCAGTTAG }\end{array}$ & 3 & $120-132$ & 0.59 & 0.25 & 0.51 \\
\hline 174 & $(\mathrm{CT})_{18}$ & $\begin{array}{l}\text { CCTGCATTCACCATCTAGGA/ } \\
\text { GGAGCCTTGCCATAACAGAT }\end{array}$ & 2 & $252-254$ & 0.50 & 0.00 & 0.38 \\
\hline 178 & $(\mathrm{CT})_{25}$ & $\begin{array}{l}\text { CCCAACTCATTTAATTCCAC/ } \\
\text { CACGTCAATCTGTTGTGAAA }\end{array}$ & 2 & $160-162$ & 0.38 & 0.00 & 0.30 \\
\hline Mean & - & - & 2.89 & & 0.55 & 0.32 & 0.48 \\
\hline
\end{tabular}

Although the primers that detected polymorphisms between accessions were different within each species, 17 were coincident, meaning that they were polymorphic among the accessions of $C$. frutescens $\mathrm{L}$. and among $C$. chinense Jacq. Thus, considering that the annealing temperature used in this study was standardized at $56^{\circ} \mathrm{C}$, these 17 primers can be used to analyze samples obtained for the 2 species. CA49 showed the largest amplitude range of alleles between the 2 species (130-132 bp for $C$. frutescens $\mathrm{L}$. and 120-128 bp for C. chinense Jacq.), and could be used to differentiate between these species. The size range of alleles was 68-254 and 68-260 bp for $C$. frutescens $\mathrm{L}$. and $C$. chinense Jacq., respectively. 
Table 3. Primers transferred from Capsicum annuum L. to C. chinense Jacq. with their motif, sequences of forward and reverse primers, allele number, size range of amplified fragment in base pairs (bp), gene diversity or expected heterozygosity $\left(H_{\mathrm{E}}\right)$, observed heterozygosity $\left(H_{\mathrm{O}}\right)$, and polymorphism information content (PIC). Brasília, DF, 2014.

\begin{tabular}{|c|c|c|c|c|c|c|c|}
\hline Primers (CA) & Motif & $\begin{array}{l}\text { Forward primer/ } \\
\text { reverse primer }\left(5^{\prime}-3^{\prime}\right)\end{array}$ & Allele No. & $\begin{array}{c}\text { Size } \\
\text { range (bp) }\end{array}$ & $\begin{array}{c}\text { Gene } \\
\text { diversity }\left(H_{\mathrm{E}}\right)\end{array}$ & $\begin{array}{c}\text { Heterozygosity } \\
\left(H_{\mathrm{O}}\right)\end{array}$ & PIC \\
\hline 1 & $(\mathrm{AG})_{19}$ & $\begin{array}{l}\text { TGGCATGGTACTTCTTAGCA/ } \\
\text { AGACACCAAGCCATCAATTA }\end{array}$ & 4 & $110-116$ & 0.65 & 0.88 & 0.58 \\
\hline 11 & $(\mathrm{CT})_{12}$ & $\begin{array}{l}\text { GTTGTTATCTCCTTTTCCCA/ } \\
\text { AAATGTTAGGAACTCACCAG }\end{array}$ & 5 & $78-94$ & 0.66 & 0.25 & 0.62 \\
\hline 19 & $(\mathrm{TC})_{12}$ & $\begin{array}{l}\text { CCGCAATGGCAGTATGATCT/ } \\
\text { CGGCTCTATCTACAACGGTG }\end{array}$ & 2 & $98-100$ & 0.22 & 0.25 & 0.19 \\
\hline 20 & $(\mathrm{AG})_{27}$ & $\begin{array}{l}\text { CCGTAAAGAAATCAAACCAC/ } \\
\text { GCATGCACACATAAACACTC }\end{array}$ & 3 & $68-90$ & 0.32 & 0.13 & 0.29 \\
\hline 24 & $(\mathrm{TC})_{11}$ & $\begin{array}{l}\text { GTTATCTCCTTTTCCCAATC/ } \\
\text { AAATGTTAGGAACTCACCAG }\end{array}$ & 5 & $84-94$ & 0.63 & 0.25 & 0.59 \\
\hline 26 & $(\mathrm{AG})_{23}$ & $\begin{array}{l}\text { CGCATATAGGCAGATCAAAT/ } \\
\text { TGACTCAAATGCTCTCTGAAA }\end{array}$ & 4 & $100-120$ & 0.73 & 0.63 & 0.68 \\
\hline 27 & $(\mathrm{CA})_{12}(\mathrm{CT})_{17} \mathrm{ATCG}(\mathrm{CT})_{9}$ & $\begin{array}{l}\text { GCAGAGGACCAGTTAGCATA/ } \\
\text { TGTTCTGAGTCCACGATGCT }\end{array}$ & 5 & $120-130$ & 0.68 & 0.80 & 0.64 \\
\hline 29 & $(\mathrm{AG})_{21}$ & $\begin{array}{l}\text { TGGGTAAAGGTACTTAGTAG/ } \\
\text { GTTGTATTGCTTTAGCTCAG }\end{array}$ & 3 & $114-128$ & 0.40 & 0.25 & 0.35 \\
\hline 41 & $(\mathrm{AG})_{27}$ & $\begin{array}{l}\text { GACATCAGTGTTCTGCAAA/ } \\
\text { ACACTGGGTATGTTGTTGTA }\end{array}$ & 3 & $152-174$ & 0.32 & 0.13 & 0.29 \\
\hline 49 & $(\mathrm{AG})_{21}$ & $\begin{array}{l}\text { CTATCTTCGCATATAGGCAG/ } \\
\text { AATCTCTGTGGCTGACTCAA }\end{array}$ & 3 & $120-128$ & 0.61 & 0.86 & 0.53 \\
\hline 54 & $(\mathrm{AG})_{25}$ & $\begin{array}{l}\text { GCAGAACATACTGAGACAGA/ } \\
\text { CTAAGTGGTCATTCGAAGAG }\end{array}$ & 2 & $136-138$ & 0.22 & 0.00 & 0.19 \\
\hline 55 & $(\mathrm{GT})_{7}(\mathrm{GA})_{23}$ & $\begin{array}{l}\text { CAGCGCTAAACAGAAGGAA/ } \\
\text { CTCTCTAAACACAAACGGCT }\end{array}$ & 2 & $114-140$ & 0.38 & 0.00 & 0.30 \\
\hline 56 & $(\mathrm{AG})_{23}$ & $\begin{array}{l}\text { CTTCGCATATAGGCAGATCA/ } \\
\text { TCTCTGTGGCTGACTCAAAT }\end{array}$ & 3 & $110-120$ & 0.54 & 0.13 & 0.45 \\
\hline 62 & $(\mathrm{AG})_{22}$ & $\begin{array}{l}\text { CGCATATAGGCAGATCAAAT/ } \\
\text { GGTCAGACTACGCTCTCTCA }\end{array}$ & 4 & $78-84$ & 0.49 & 0.38 & 0.46 \\
\hline 66 & $(\mathrm{TC})_{18}$ & $\begin{array}{l}\text { CAACCCATACAAATCATCCA/ } \\
\text { AGGGTTGAGCAATTCATAGA }\end{array}$ & 2 & $122-124$ & 0.38 & 0.00 & 0.30 \\
\hline 67 & $(\mathrm{GA})_{25}$ & $\begin{array}{l}\text { CCGAGAAAATGCACACAA/ } \\
\text { TGACATACTCTTCCTACAGCTA }\end{array}$ & 4 & $90-104$ & 0.68 & 0.13 & 0.62 \\
\hline 75 & $(\mathrm{TC})_{26}$ & $\begin{array}{l}\text { CAACACTAAGTGGTCATTCG/ } \\
\text { CTGAGACAGAAATTCTTGCT }\end{array}$ & 2 & $136-138$ & 0.38 & 0.00 & 0.30 \\
\hline 79 & $(\mathrm{TC})_{26}$ & $\begin{array}{l}\text { CACTGGGTATGTTGTTGTAA/ } \\
\text { CCGTAAAGAAATCATACCAC }\end{array}$ & 3 & $90-112$ & 0.32 & 0.13 & 0.29 \\
\hline 80 & $(\mathrm{TC})_{20}(\mathrm{TG})_{7}$ & $\begin{array}{l}\text { CTCAAGTGTGCCAGGTGATT/ } \\
\text { GAGAGACAGGAAGAGACGTACA }\end{array}$ & $\mathrm{A}^{2}$ & $106-122$ & 0.22 & 0.00 & 0.19 \\
\hline 88 & $(\mathrm{AG})_{22}$ & $\begin{array}{l}\text { AATGGATGTTCCCTTGCTTT/ } \\
\text { CAACTGATCAACCATTCCGT }\end{array}$ & 4 & $150-162$ & 0.67 & 0.38 & 0.61 \\
\hline 94 & $(\mathrm{GT})_{5}(\mathrm{AG})_{22}$ & $\begin{array}{l}\text { ACACAAAATGTCCCCGAA/ } \\
\text { CCATTGACAAGGACAATTCT }\end{array}$ & 2 & $112-114$ & 0.22 & 0.00 & 0.19 \\
\hline 95 & $(\mathrm{AG})_{20}$ & $\begin{array}{l}\text { CGCATATAGGCAGATCAAAT/ } \\
\text { AATCTCTGTGGCTGACTCAA }\end{array}$ & 4 & $108-120$ & 0.66 & 0.43 & 0.62 \\
\hline 96 & $(\mathrm{AG})_{23}$ & $\begin{array}{l}\text { CGCATATAGGCAGATCAAAT/ } \\
\text { AATCTCTGTGGCTGACTCAA }\end{array}$ & 4 & $108-120$ & 0.70 & 0.88 & 0.64 \\
\hline 98 & $(\mathrm{AG})_{20}$ & $\begin{array}{l}\text { CGAGCTAATGGCATGGTACT/ } \\
\text { TCAGACACCAAGCCATCAAT }\end{array}$ & 3 & $122-128$ & 0.55 & 1.00 & 0.46 \\
\hline 99 & $(\mathrm{AG})_{23}$ & $\begin{array}{l}\text { TTCGCATATAGGCAGATCAA/ } \\
\text { AATCTCTGTGGCTGACTCAA }\end{array}$ & 3 & $120-130$ & 0.54 & 0.13 & 0.45 \\
\hline 101 & $(\mathrm{AG})_{17}$ & $\begin{array}{l}\text { TGATTAGGGCGTAGGGTTTA/ } \\
\text { TATTCCTCTTCACCTCCGCT }\end{array}$ & 2 & $130-134$ & 0.22 & 0.00 & 0.19 \\
\hline 105 & $(\mathrm{AG})_{20}$ & $\begin{array}{l}\text { CTTCGCATATAGGCAGATCA/ } \\
\text { GGCTGACTCAAATGCTCTCT }\end{array}$ & 3 & $112-120$ & 0.54 & 0.13 & 0.45 \\
\hline 130 & $(\mathrm{AG})_{44}$ & $\begin{array}{l}\text { TGCATACGCTGGTGTGTC/ } \\
\text { TTCAGCTGTGGTTATGGG }\end{array}$ & 3 & $112-126$ & 0.55 & 0.25 & 0.46 \\
\hline 131 & $(\mathrm{AG})_{26}$ & $\begin{array}{l}\text { AAATGCACACAAAAACAC/ } \\
\text { AATATGACCACATTTGTG }\end{array}$ & 3 & $114-130$ & 0.66 & 0.14 & 0.59 \\
\hline
\end{tabular}

Continued on next page 


\begin{tabular}{|c|c|c|c|c|c|c|c|}
\hline Primers (CA) & Motif & $\begin{array}{l}\text { Forward primer/ } \\
\text { reverse primer }\left(5^{\prime}-3^{\prime}\right)\end{array}$ & Allele No. & $\begin{array}{c}\text { Size } \\
\text { range (bp) }\end{array}$ & $\begin{array}{c}\text { Gene } \\
\text { diversity }\left(H_{\mathrm{E}}\right)\end{array}$ & $\begin{array}{l}\text { Heterozygosity } \\
\left(H_{\mathrm{O}}\right)\end{array}$ & PIC \\
\hline 136 & $(A G)_{16}$ & $\begin{array}{l}\text { GTGGACTAACAGACTCAACG/ } \\
\text { CTGATGATGCAGAACATGAT }\end{array}$ & 2 & $150-156$ & 0.47 & 0.00 & 0.36 \\
\hline 140 & $(\mathrm{GA})_{12}$ & $\begin{array}{l}\text { GTGTGTCTGTGTGCATGAGC/ } \\
\text { TTCAGCTGTGGTTATGGGA }\end{array}$ & 3 & $90-114$ & 0.55 & 0.38 & 0.46 \\
\hline 159 & $(\mathrm{AG})_{19}$ & $\begin{array}{l}\text { GCAGAAAATGGGTAAAGGTA/ } \\
\text { ATTGCTTTAGCTCAGAATGG }\end{array}$ & 4 & $110-120$ & 0.55 & 0.25 & 0.51 \\
\hline 167 & $(\mathrm{TC})_{24}$ & $\begin{array}{l}\text { CATTCTCTTCTTCTCAAACG/ } \\
\text { CACGTCAATCTGTTGTGAAA }\end{array}$ & 5 & $80-130$ & 0.66 & 0.63 & 0.62 \\
\hline 172 & $(\mathrm{TC})_{9} \operatorname{GCTA}(\mathrm{TC})_{14}(\mathrm{CA})_{13}$ & $\begin{array}{l}\text { ATGTTCTGAGTCCACGATGC/ } \\
\text { CTTAGCAGAGGGCCAGTTAG }\end{array}$ & 3 & $88-132$ & 0.36 & 0.14 & 0.33 \\
\hline 174 & $\left(\mathrm{CT}_{18}\right)$ & $\begin{array}{l}\text { CCTGCATTCACCATCTAGGA/ } \\
\text { GGAGCCTTGCCATAACAGAT }\end{array}$ & 5 & $160-260$ & 0.62 & 0.29 & 0.59 \\
\hline 178 & $(\mathrm{CT})_{25}$ & $\begin{array}{l}\text { CCCAACTCATTTAATTCCAC/ } \\
\text { CACGTCAATCTGTTGTGAAA }\end{array}$ & 5 & $160-200$ & 0.57 & 0.38 & 0.54 \\
\hline Mean & - & - & 3.30 & & 0.50 & 0.29 & 0.44 \\
\hline
\end{tabular}

A total of 55 alleles were identified from the 19 polymorphic SSR loci among accessions of $C$. frutescens $\mathrm{L}$. The number of alleles per locus was 2-5, with a mean of 2.89. PIC ranged from 0.30-0.65. Loci showing the highest PIC values included CA 62, CA 19, and CA 26 with PIC values of $0.60,0.63$, and 0.65 , respectively. In 10 of the 19 loci (CA 20, CA 29, CA 39, CA 41, CA 49, CA 79, CA 88, CA 167, CA 174, and CA 178), the observed heterozygosity $\left(H_{\mathrm{O}}\right)$ was 0 , corresponding to $100 \%$ homozygous individuals, and in 3 loci (CA 19, CA 52 , and CA 96), $H_{\mathrm{O}}$ was equal to 1 , corresponding to $100 \%$ heterozygous individuals.

The number of alleles identified among the sampled individuals of $C$. chinense Jacq. was 119 , ranging from 2-5 alleles per locus and an average of 3.30. Only 8 of the 36 loci (CA54, CA 55, CA 66, CA 75, CA 80, CA 94, CA 101, and CA 136) presented $H_{\mathrm{O}}$ equal to 0 and 5 of the 36 loci (CA 1, CA 27, CA 49, CA 96, and CA 98) had values of $H_{\mathrm{O}}$ higher than the expected heterozygosity $\left(H_{\mathrm{E}}\right)$. The loci analyzed in $C$. chinense Jacq. showed PIC variation of 0.19-0.68, with high level (greater than 0.5) of polymorphic information, 0.62 for CA 11, CA 67, CA 95, and CA 167 and 0.64 for CA 27 and CA 96 and 0.68 for CA 26.

\section{DISCUSSION}

Transferability of microsatellite primers for species within the same genus has been reported previously, such as in Euterpe, E. edulis to E. oleracea, the 7 loci tested presenting 100\% success (Oliveira et al., 2010) and Anacardium, A. occidentale, to A. humile, with transferability of all 11 loci analyzed (Cota et al., 2012). Furthermore, rates of polymorphism higher than those observed in this study were obtained by Ince et al. (2010a), who detected polymorphisms between the species $C$. annuum $\mathrm{L}$. and $C$. frutescens $\mathrm{L}$., with 34 microsatellite primer pairs among the 45 pairs tested. This corresponds to a rate of $75.5 \%$ of polymorphism and 2.5-4.6 times the rate of polymorphism obtained in the present study for $C$. chinense Jacq. and $C$. frutescens L., respectively.

The average PIC values obtained for $C$. frutescens $\mathrm{L}$. (0.48) and $C$. chinense Jacq. (0.44) were higher compared to those observed by Nagy et al. (2007) in 14 accessions of $C$. annuum L. evaluated using 2 microsatellite markers (EPMS $=0.30$ and GPMS $=0.27)$ and lower when the author evaluated 33 genotypes of 8 domesticated and wild species of Capsicum $(\mathrm{EPMS}=0.67$ and GPMS $=0.68)$. This higher level of interspecific polymorphism was 
observed likely because the materials used were extremely diverse. Polymorphic microsatellite markers for mapping in Capsicum developed by Lee et al. (2004) from genomic libraries showed a high PIC value (0.75), which is twice the value obtained by the same authors using primers designed based on GenBank data (0.38).

The difference between the numbers of alleles in individuals sampled for $C$. chinense Jacq. and $C$. frutescens $\mathrm{L}$. was clearly observed in this study. The smaller number of alleles for C. frutescens L., as well as the higher rate of homozygosity found, may reflect the relatively lower genetic variability shown by this species compared with other species in the genus. Capsicum frutescens L. showed the most conserved morphology, with fewer shapes, sizes, and colors of fruits compared to C. annuum L., C. chinense Jacq., and C. baccatum L. (DeWitt and Bosland, 2009). In contrast, $C$. chinense Jacq. can be distinguished based on its extensive morphological variability, expressed in diverse forms and colors of fruits, which are typically very spicy and aromatic (Ribeiro et al., 2008).

This study describes microsatellite primers showing acceptable amplification and polymorphism for the species $C$. frutescens $\mathrm{L}$. and $C$. chinense Jacq. Countries in the "new world", including those in South America, regard these species as being economically and socially important (Moses et al., 2014), and may show the highest benefit from our results. In these countries, the planning and implementation of germplasm conservation and breeding programs are limited by the lack of knowledge regarding the genetic structure and diversity of C. frutescens L. and C. chinense Jacq. (Moses et al., 2014). Thus, the primers described in this study can be used in studies of diversity and phylogeny.

Other studies have reported a considerable number of microsatellite primers evaluated for different sets comprising Capsicum species, including C. frutescens $\mathrm{L}$. and C. chinense Jacq. [Lee et al., 2004 (76 primers), Nagy et al., 2007 (157 primers), Ince et al., 2010b (45 primers)]. Our study offers additional primers, evaluated specifically for these 2 species (19 primers for $C$. frutescens $\mathrm{L}$., assessed for 4 accessions, and 36 for $C$. chinense Jacq., analyzed for 8 accessions). These primers were reliable and polymorphic within accessions of a Brazilian collection, including accessions with typically wild characteristics such as CNPH 4353 ( $C$. frutescens L.) and CNPH 4315 (C. chinense Jacq.), the latter collected in the Amazon region, a diversity center for $C$. chinense Jacq. (Moses et al., 2014).

Microsatellite primers showing polymorphism among wild and cultivated genotypes enable further studies on the structure of these species. Moreover, one of the primers described (CA49) was able to differentiate between $C$. frutescens $\mathrm{L}$. and $C$. chinense Jacq., and may be useful to the classification of the Germplasm Bank of Embrapa and, possibly, in other studies of the Brazilian germplasm. The primers can also be used for diversity studies, allowing for the selection of the most divergent genotypes with potential for heterosis gains through hybridization (Moses et al., 2014). Recently, we used the same microsatellite primers to obtain a core collection of $C$. frutescens $\mathrm{L}$. that included 13 accessions, representing 77 and $81 \%$ of the alleles and the morphologic categories, respectively, found in the complete collection (104 accessions) (Carvalho SIC, unpublished results). These data reinforce the potential impact of this study, particularly for further studies of the species $C$. frutescens L. and C. chinense Jacq.

In summary, the CA microsatellite markers developed for $C$. annuum L. were most often transferable and polymorphic for the species $C$. frutescens L. and C. chinense Jacq. A set of SSRs was presented for each of these species, which may be valuable for germplasm identification as well as for further genetic, diversity, and phylogeny studies. 


\section{ACKNOWLEDGMENTS}

Research supported by Embrapa (Empresa Brasileira de Pesquisa Agropecuária), project \#02.12.02.007.00.00, and Conselho Nacional de Desenvolvimento Científico e Tecnológico (CNPq), project \#402121/2008-2.

\section{REFERENCES}

Bianchetti LB and Carvalho SIC (2005). Subsídios à coleta de germoplasma de espécies de pimentas e pimentões de gênero Capsicum (Solanaceae) In: Fundamentos para a coleta de germoplasma vegetal (Walter BMT and Cavalcanti TB, eds.). Embrapa Recursos Genéticos e Biotecnologia, Brasília, 355-385.

Bravo JP, Hoshino AA, Angelici CMLCD, Lopes CR, et al. (2006). Transferability and use of microsatellite markers for the genetic analysis of the germplasm of some Arachis section species of the genus Arachis. Genet. Mol. Biol. 29: 516-524.

Buso GSC, Brondani RPV, Amaral ZPS, Reis AMM, et al. (2000). Desenvolvimento de primers ssr para análise genética de pimentas e pimentões (Capsicum spp.) utilizando biblioteca genômica enriquecida. Bol. Pesq. Embrapa Rec. Genet. e Biotecnol. 15: 1-27.

Carvalho SIC, Bianchetti LB, Ribeiro CSC and Lopes CA (2006). Pimentas do gênero Capsicum no Brasil. Doc. Embrapa Hort. 94: 1-49.

Ciampi AY, Azevedo VCR, Gaiotto FA, Ramos AC, et al. (2008). Isolation and characterization of microsatellite loci for Hymenaea courbaril and transferability to Hymenaea stigonocarpa, two tropical timber species. Mol. Ecol. Resour. 8: 1074-1077.

Cota LG, Moreira PA, Menezes EV, Gomes AS, et al. (2012). Transferability and characterization of simple sequence repeat markers from Anacardium occidentale to A. humile (Anacardiaceae). Genet. Mol. Res. 11: 4609-4616.

Creste S, Tulmann Neto A and Figueira A (2001). Detection of single sequence repeat polymorphisms in denaturing polyacrylamide sequencing gels by silver staining. Plant Mol. Biol. Rep. 19: 299-306.

DeWitt D and Bosland PW (2009). The complete chile pepper book: a gardener's guide to choosing, growing, preserving, and cooking. 1st edn. Timber Press, London.

Ferreira ME and Grattapaglia D (1998). Introdução ao uso de marcadores moleculares em análise genética. 1st edn. Embrapa Recursos Genéticos e Biotecnologia, Brasília.

Ince AG, Karaca M and Onus AN (2010a). Genetic relationships within and between Capsicum species. Biochem. Genet. 48: 83-95.

Ince AG, Karaca M and Onus AN (2010b). Polymorphic microsatellite markers transferable across Capsicum species. Plant Mol. Biol. Rep. 28: 285-291.

IPGRI (International Plant Genetic Resources Institute) (1995). Descriptors for Capsicum (Capsicum spp.). IPGRI, Rome.

Lee JM, Nahm SH, Kim YM and Kim BD (2004). Characterization and molecular genetic mapping of microsatellite loci in pepper. Theor. Appl. Genet. 108: 619-627.

Lincoln SE, Daly MJ and Lander ES (1991). PRIMER Software. Available from E. Lander, Whitehead Institute, Cambridge, MA.

Liu KJ and Muse SV (2005). PowerMarker: an integrated analysis environment for genetic marker analysis. Bioinformatics 21: $2128-2129$.

Lorieux M, Ndjiondjop MN and Ghesquière A (2000). A first interespecific Oryza sativa x Oryza glaberrima microsatellitebased genetic linkage map. Theor. Appl. Genet. 100: 593-601.

Moses M, Umaharan P and Dayanandan S (2014). Microsatellite based analysis of the genetic structure and diversity of Capsicum chinense in the Neotropics. Genet. Resour. Crop Evol. 61: 741-755.

Nagy I, Stágel A, Sasvári Z, Röder M, et al. (2007). Development, characterization, and transferability to other Solanaceae of microsatellite markers in pepper (Capsicum annuиm L.). Genome 50: 668-688.

Oliveira MSP, Santos JB, Amorim EP and Ferreira DF (2010). Variabilidade genética entre acessos de açaizeiro utilizando marcadores microssatélites. Cienc. Agrotec. 34: 1253-1260.

Pickersgill B (1988). The genus Capsicum: a multidisciplinary approach to the taxonomy of cultivated and wild plants. Biologisches Zentralblatt 107: 381-389.

Reifschneider FJB, Oliveira AB, Silva AM, Lopes CA, et al. (2000). Capsicum - pimentas e pimentões no Brasil. 1st edn. Embrapa Comunicação para a Transferência de Tecnologia, Brasília.

Ribeiro CSC, Lopes CA, Carvalho SIC, Henz GP, et al. (2008). Pimentas Capsicum. Embrapa Hortaliças, Brasília. 
Ritschel PS, Lins TCL, Tristan RL, Buso GSC, et al. (2004). Development of microsatellite markers from an enriched genomic library for genetic analysis of melon. BMC Plant Biol. 4: 9-24.

Yi G, Lee JM, Lee S, Choi D, et al. (2006). Exploitation of pepper EST-SSRs and an SSR-based linkage map. Theor. Appl. Genet. 114: 113-130.

Zucchi MI, Brondani RPV, Pinheiro JB, Chaves LJ, et al. (2003). Genetic structure and gene flow in Eugenia dysenterica DC in the Brazilian Cerrado utilizing SSR markers. Genet. Mol. Biol. 26: 449-457. 